\title{
Transverse beam tails due to inelastic scattering
}

\author{
H. Burkhardt, I. Reichel,* and G. Roy \\ CERN, CH-1211 Geneva, Switzerland \\ (Received 7 December 1999; published 7 September 2000)
}

\begin{abstract}
Non-Gaussian beam tails producing low beam lifetimes and background to the experimental detectors can be a serious performance limitation in colliding beam facilities. We describe simulations and measurements of non-Gaussian beam tails performed on the $e^{+} e^{-}$collider LEP that revealed the importance of inelastic particle scattering as launching processes of particles to large amplitudes.
\end{abstract}

PACS numbers: 29.20.Dh, 29.27.Fh, 41.85.Ew, 41.85.Si

\section{INTRODUCTION}

The general importance of the beam-beam interaction and non-Gaussian beam tails for the performance of highenergy colliding beam facilities is well established $[1,2]$. The strength of the beam-beam interaction is quantified by the beam-beam tune shift parameters, usually noted as $\xi_{y}$ for the vertical plane and $\xi_{x}$ for the horizontal plane. A strong beam-beam interaction results in increased beam sizes limiting the maximum beam-beam parameter $\xi$ to values of 0.04 to 0.08 for $e^{+} e^{-}$colliders such that the increase in luminosity becomes linear with beam current, rather than quadratic as expected for constant beam sizes. This is called the (first) beam-beam limit, and colliders are generally designed to operate with beam currents such that saturation of the beam-beam parameter is reached. Further increasing the beam current results in the development of non-Gaussian beam tails leading to low beam lifetimes or unacceptable background to the experimental detectors; this ultimately limits the maximum beam currents that can safely be collided and is sometimes called the second beam-beam limit [3].

Non-Gaussian tails in the beam distributions have been studied extensively in LEP [4] and, in fact, limited the beam currents that could be collided at medium beam energies, around $45.6 \mathrm{GeV}$ per beam. Various simulation codes have been used to predict the effects of the beambeam interaction on colliding beams in LEP [5-7]. The quantitative measurements using tail scans in LEP showed much more extended tails than predicted. In particular, it was observed that, on a low but still well measurable level, extended non-Gaussian tails were already present in the absence of the beam-beam interaction [8-10], i.e., with a single beam or separated beams. General aspects of beam dynamics in LEP have recently been summarized in [11]. The performance and beam-beam behavior observed in LEP at different beam energies is described in $[12,13]$.

In this paper we describe the mechanism that launches off-momentum particles originating from inelastic scatter-

\footnotetext{
*Also at III. Physikalisches Institut, RWTH Aachen, Germany. Present address: SLAC, Stanford, California.
}

ing processes into extended beam tails and describe the main observations and recent simulations supporting the importance of incoherent scattering processes in the formation of beam tails.

\section{TAIL SCANS}

The technique of tail scans as used in LEP was proposed by Zholents [14]. Dedicated collimators are located in a straight section (IP5), where the nominal dispersion is zero in both planes, and far away from the LEP experiments. A collimator is moved closer to the beam in small steps of the order of $5 \%$ to $10 \%$ of the standard deviation of the beam distribution. At each step the loss rate at the collimator is measured using $p$ - $i$-n-diode-type beam loss monitors which were developed at DESY [15]. The loss monitors are attached to the collimator as sketched in Fig. 1.

The loss monitors are calibrated with beam lifetime measurements from the beam current transformer (BCT) at low beam lifetimes and correspondingly very high loss rates. The procedure is described in detail in [4]. It should be noted that the beam loss monitors are much more sensitive than the beam lifetime measurement from the $\mathrm{BCT}$

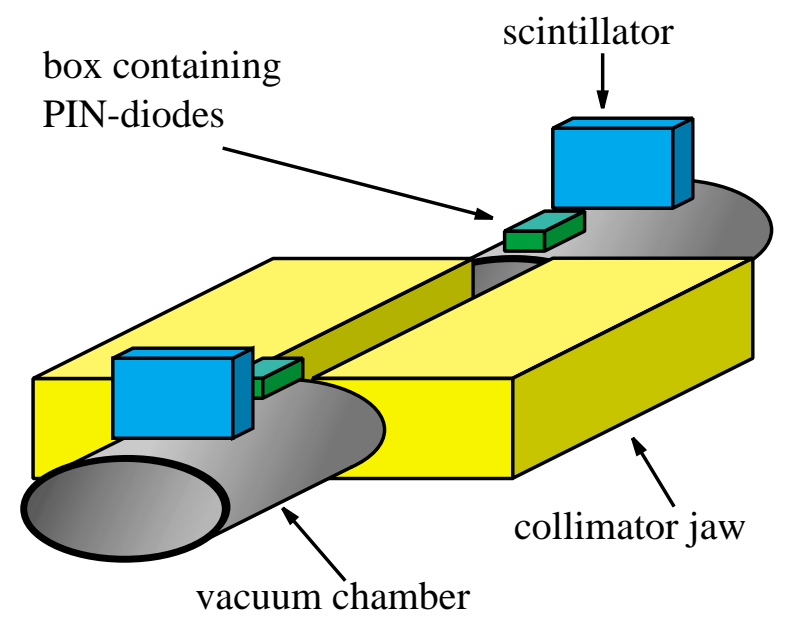

FIG. 1. (Color) Schematic drawing of a horizontal collimator with the $p$ - $i$ - $n$-diode beam loss monitors and the scintillators on both sides. For vertical collimators the installation is turned by $90^{\circ}$. The scintillators are used only for on-line measurements. 
for long beam lifetimes. The range of sensitivity of the beam loss monitors extends from several thousand hours to about $0.1 \mathrm{~h}$, using two sets of $p-i-n$ diodes of different sizes.

The horizontal beam tails measured for single beam and colliding beams with moderate beam-beam tune shift looked very similar $[8,9]$. A sharp increase in loss rate is observed at about five standard deviations of the beam distribution, as expected from the horizontal emittance for a Gaussian beam. At a low level, corresponding to a beam lifetime of a few hundred to a few thousand hours, broad non-Gaussian tails were recorded, rather independent of machine parameters such as tune or chromaticity, and scattering processes were investigated as a possible cause.

Scattering angles from elastic scattering on residual gas were found to be rather small (probabilities corresponding to $47 \mathrm{~h}$ for $10 \mu \mathrm{rad}$ and $4700 \mathrm{~h}$ for $100 \mu \mathrm{rad}$ ); i.e., scattering angles are mainly comparable to the divergence of the beam.

\section{INELASTIC SCATTERING}

Inelastic scattering, on the contrary, was found to produce rather extended tails, with a mechanism similar to the excitation process originating in the emission of synchrotron radiation which, together with radiation damping, leads to the equilibrium emittance in a storage ring. Instead of the emission of a photon, we consider an inelastic scattering process in a region where the dispersion function is nonzero. The energy of the particle being changed, its reference orbit, is shifted with respect to its original orbit and the particle starts a betatron oscillation around the new reference orbit (see Fig. 2). Depending on the energy change in the scattering process, the dispersion and betatron func-

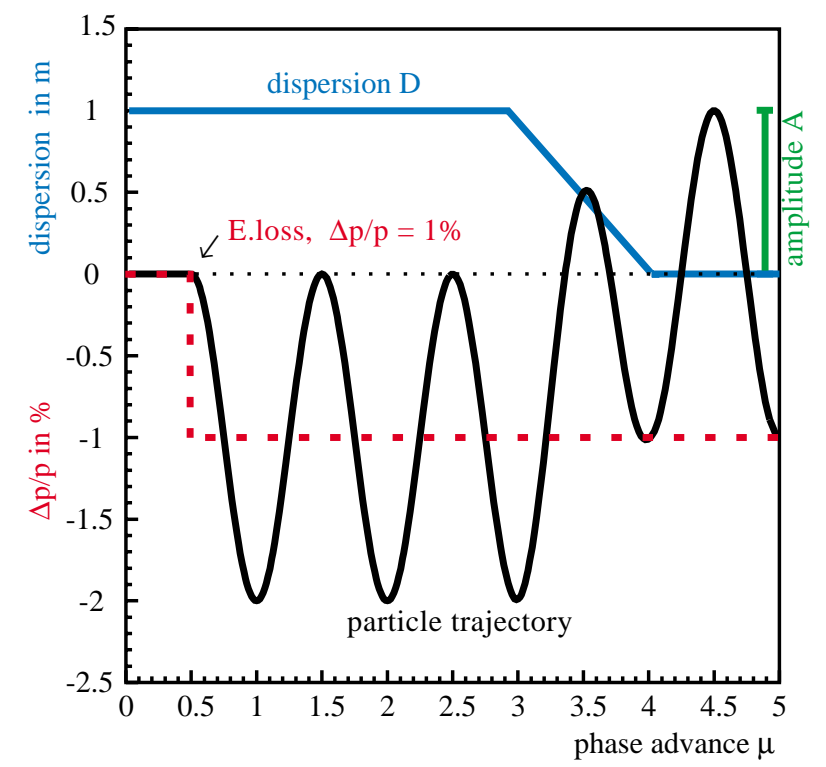

FIG. 2. (Color) Dispersion and amplitude of a particle versus the phase advance for a particle before and after inelastic scattering. tions at the location of the scattering, the betatron oscillation amplitude, can be large enough to place the particle far in the tails of the transverse distribution. The main inelastic scattering process contributing to the horizontal single beam tails in LEP is the Compton scattering on thermal photons from the blackbody radiation of the beam pipe. The contribution from the inelastic scattering on residual gas is about 1 order of magnitude smaller because of the good vacuum in LEP with a residual pressure of the order of $10^{-10}$ Torr [16]. However, for a relative energy loss of the order of $10^{-2}$, as is relevant here, the energy spectra and resulting tail distributions of the Compton scattering and beam-gas scattering processes are not too different [4], such that most of the general features discussed here for LEP should also apply to lower energy machines where the beam-gas scattering dominates.

In LEP the vertical dispersion $\left(D_{y}\right)$ is zero by design, and the residual values arising from orbit, alignment errors, and other perturbations are much smaller than the nominal horizontal dispersion; typical rms values along the ring are $\left\langle D_{x}\right\rangle \approx 1 \mathrm{~m}$ and $\left\langle D_{y}\right\rangle \approx 5 \mathrm{~cm}$. The betatron functions, on the other hand, are comparable in both planes with an average value $\bar{\beta} \approx 100 \mathrm{~m}$ for the lattices currently used for physics production.

The normalized amplitude of the oscillation originating in the inelastic scattering process is proportional to $(\Delta p / p)(D / \sqrt{\beta})$. It is therefore expected to generate much more extended tails in the horizontal plane. Typical numbers for LEP are $D_{x} / \sqrt{\beta_{x}} \approx 0.1 \sqrt{m}$ and $D_{y} / \sqrt{\beta_{y}} \approx$ $0.005 \sqrt{m}$.

At the interaction point, the beam-beam bremsstrahlung, another strong inelastic scattering process, also called low angle radiative Bhabha scattering, constitutes a large localized source of off-momentum particles. Tracking studies with DIMAD [17] have shown that the beam-beam bremsstrahlung does create significant vertical beam tails even in the absence of residual dispersion at the interaction point. We suspect that this is related to the large local chromaticity generated by the low-beta quadrupoles near the interaction point. Nonlinearities in the lattice at large amplitudes and energy offsets enhance these effects.

\section{SIMULATION TECHNIQUE}

The Monte Carlo generator described in [18] was used to simulate Compton scattering on thermal photons of the blackbody radiation of the vacuum chamber. New procedures, based on standard Monte Carlo techniques including inverse transform and rejection, have been written for the elastic and inelastic beam-gas scattering and are described in some detail in [4]. For the beam-beam bremsstrahlung, simple routines that reproduce approximately the energy spectrum of the program described in [19] have been developed. These generators were then implemented in the tracking code DIMAD [17]. 
In order to simulate the tail scan experiments, further modifications have been made to the tracking routines of DIMAD. The scattering probabilities are adjusted such that most particles have scattered after the first turn. Those which have not scattered after the first turn are discarded. The scattering processes are then disabled and the particles are tracked, using the usual methods, for at least one damping time during which the maximum amplitude of each particle at the scanning collimator is recorded. The beam loss rate is then estimated by counting the number of particles for which the maximum amplitude was larger than the assumed position of the scanning collimator.

\section{MEASURED AND SIMULATED HORIZONTAL TAILS}

The tails in the horizontal distribution of a single positron beam were first measured (at a beam energy of $80.5 \mathrm{GeV}$ ) with all collimators open to prephysics high energy settings ("RAMP\&SQUEEZE"), corresponding to a normalized physical aperture of at least $\sqrt{A_{x}}=$ $3.2 \times 10^{-3} \sqrt{m}$ or $17 \sigma_{x}$, assuming a horizontal emittance of $38 \mathrm{~nm}$.

A horizontal aperture collimator (COLH.QS1B.R4) in a region without dispersion was then closed to $8.5 \sigma_{x}$ or $\sqrt{A_{x}}=1.7 \times 10^{-3} \sqrt{m}$, which resulted in a reduction of the extended tails.

Finally, a group of four collimators, located in a region of high horizontal dispersion and designed to shield

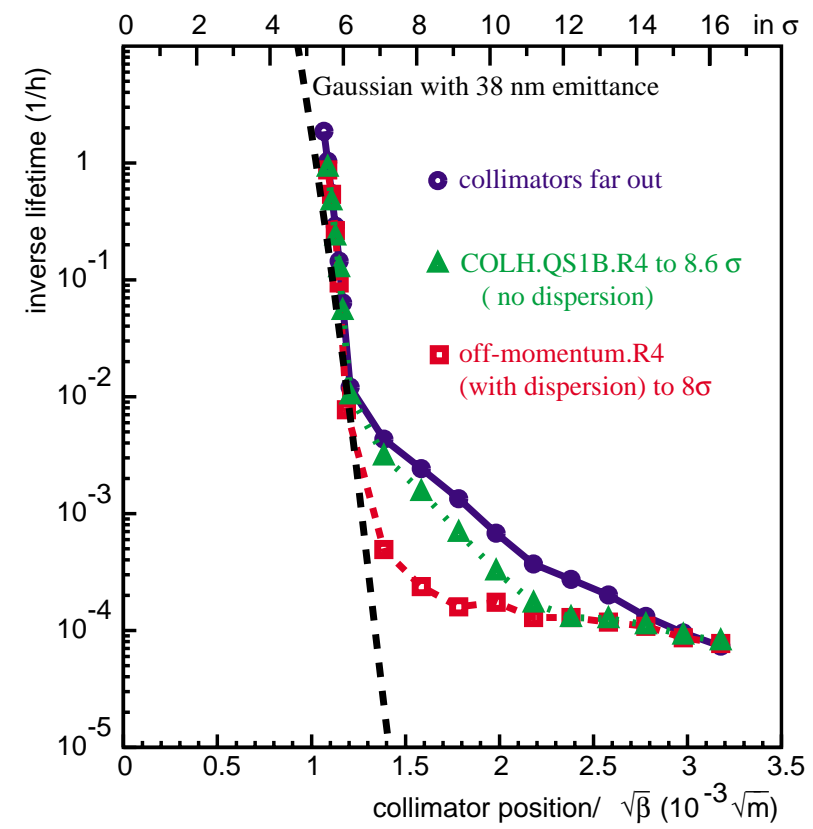

the experiments from off-momentum particles at different phases, was closed to $8 \sigma_{x}$ or $\sqrt{A_{x}}=1.6 \times 10^{-3} \sqrt{m}$ with the horizontal aperture collimator opened again; the result was a very significant reduction of the extended tails thereby confirming the off-momentum nature of these beam tails.

The results of the measurements are shown on the left side of Fig. 3. The right side of Fig. 3 shows simulation results from DIMAD for scattering on thermal photons and the same collimator settings as in the measurements.

The measurement and the simulation agree well within errors. The overall normalization uncertainty in the measurements is estimated to be $30 \%$. If beam-gas scattering is included in the simulation the loss rates increase by $10 \%$ to $30 \%$ depending on vacuum conditions. The effectiveness of off-momentum collimation observed in the data is well reproduced in the simulation and demonstrates the importance of inelastic scattering as a process to launch particles into extended non-Gaussian beam tails.

\section{MEASURED AND SIMULATED VERTICAL TAILS}

In the vertical plane, we found that the observed beam tails at moderate beam-beam tune shifts can be largely attributed to the beam-beam bremsstrahlung at the interaction point. This can be seen in Fig. 4. The measurements are shown by dots connected by solid lines. The data were taken in LEP at a beam energy of $45.6 \mathrm{GeV}$

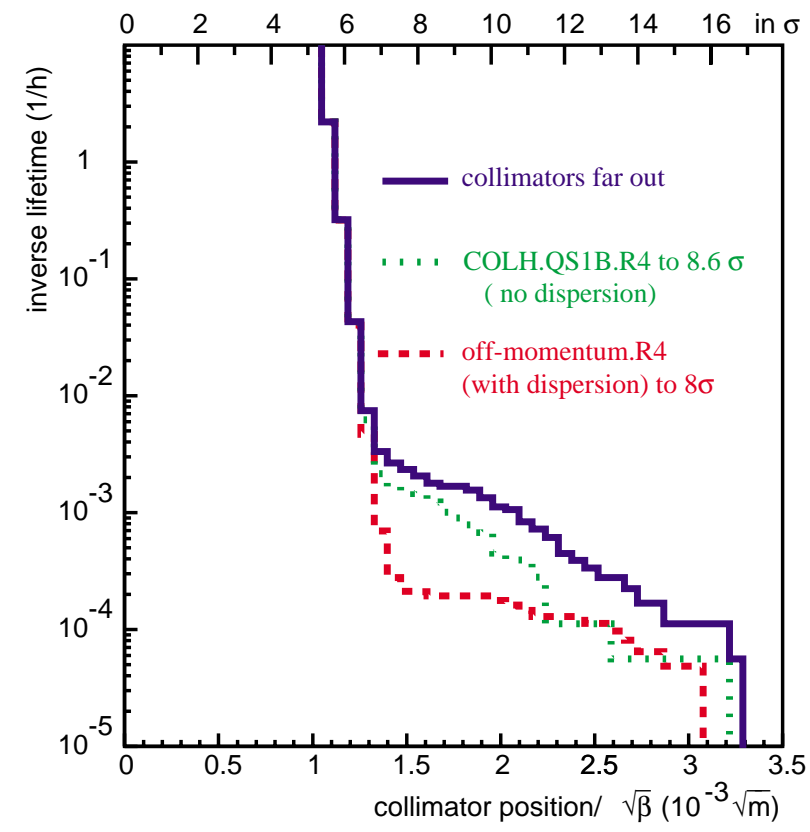

FIG. 3. (Color) Measurement (left) and simulation (right) of the horizontal beam tails for a beam energy of $80.5 \mathrm{GeV}$ and for different collimator settings. The simulation is the result of tracking particles after scattering on thermal photons. The collimator position is given in invariant amplitude on the lower scale and in number of standard deviations of the beam distribution-assuming a horizontal emittance of $38 \mathrm{~nm}$ - on the upper scale. Several data points have been combined in the measurement in order to reduce the statistical error, now of the order of the size of the symbols on the plot. The expected losses for a Gaussian beam with a $38 \mathrm{~nm}$ horizontal emittance is shown on top of the data. 

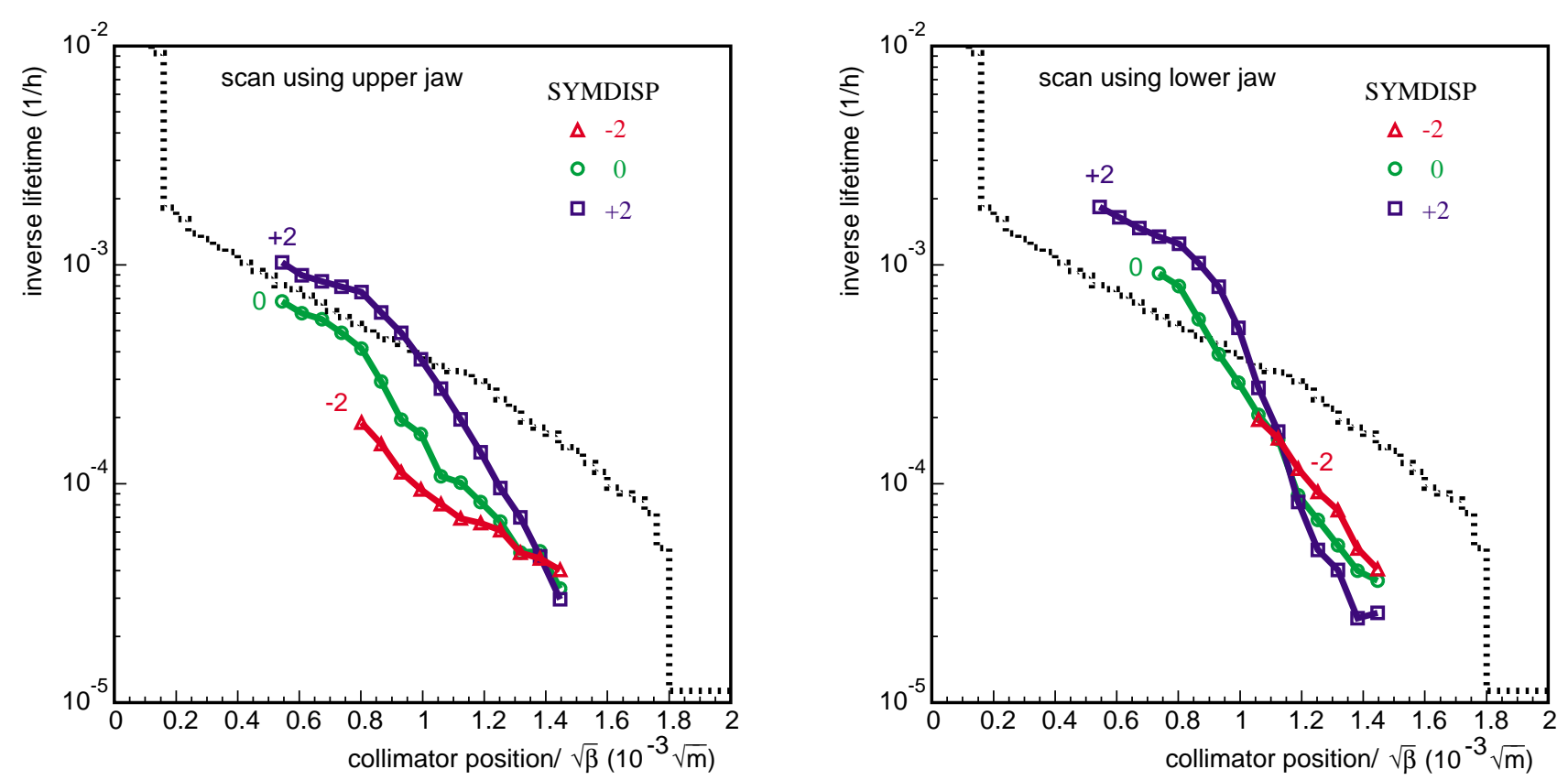

FIG. 4. (Color) Vertical tails for different vertical dispersions at the IPs measured in 1997 at $45.6 \mathrm{GeV}\left(90^{\circ} / 60^{\circ}\right.$ lattice) for the upper (left) and the lower (right) jaw with $\xi_{y} \approx 0.025$. The dotted line gives the simulation results for no dispersion at the IP and $\xi_{y}=0.025$.

for colliding beams and a vertical beam-beam tune shift $\xi_{y} \approx 0.025$. The dotted histogram gives the simulation results in the absence of vertical dispersion at the interaction point and using nominal magnet strengths. For the measurements, we expect a small residual vertical dispersion of a few millimeters. Using vertical orbit bumps (controlled by a control system variable called "SYMDISP") we changed the vertical dispersion by wellknown amounts. A trim of SYMDISP by \pm 2 units as shown in Fig, 4 corresponds to a change of dispersion at the interaction point of $D_{y}^{*}= \pm 4.4 \mathrm{~mm}$ and of $\mp 100 \mathrm{~mm}$ at the collimator used for the tail scan. The lowest level of tails was observed for SYMDISP $=-2$, possibly because of a cancellation of residual and artificially introduced dispersion.

In Fig. 4, the measurements fall below the simulation for amplitudes above $1.2 \times 10^{-3} \sqrt{m}$. This can be attributed to differences in aperture between measurement and simulation. For the measurements, both the dynamic and physical aperture, restricted by collimators to screen the experiments from excessive synchrotron light, were about $\sqrt{A_{y}}=1.5 \times 10^{-3} \sqrt{m}$. The vertical aperture for the rather idealized simulation instead extended to about $\sqrt{A_{y}}=2 \times 10^{-3} \sqrt{m}$.

For the simulation, our emphasis has been on an adequate simulation of the inelastic scattering to demonstrate the importance of inelastic scattering processes. Possible further improvements would be a more realistic simulation of machine errors, full implementation of the beam-beam interaction for head-on collisions, as well as separated encounters and tracking of many more particles over more turns. Ultimately, this should allow one to fully account for the increase of non-Gaussian tails observed for strong beam-beam interaction [13] and to actually predict the maximum useful beam current that can be collided, known as the second beam-beam limit.

\section{CONCLUSION}

We have studied transverse beam tails in LEP. The studies revealed the importance of inelastic particle scattering processes to launch particles into rather extended non-Gaussian tails. For the horizontal plane with long sections of high dispersion, scattering with particles outside the beam dominates (beam-gas or Compton scattering on blackbody photons). In the vertical plane, the dominant inelastic scattering process is the beam-beam bremsstrahlung.

[1] J. T. Seeman, in Nonlinear Dynamic Aspects of Particle Accelerators, edited by J. Jowett, M. Month, and S. Turner, Lecture Notes in Physics Vol. 247 (Springer-Verlag, Berlin, Heidelberg, New York, 1985), pp. 121-153.

[2] A. B. Temnykh et al., in Proceedings of the 3rd Advanced Beam Dynamics Workshop on Beam-Beam Effects in Circular Colliders, Novosibirsk, 1989 (Institute of Nuclear Physics, Novosibirsk, 1989), pp. 5-145.

[3] M. Furman, in Proceedings of the 14th Advanced ICFA Beam Dynamics Workshop on Beam Dynamics Issues for $e^{+} e^{-}$Colliders, Frascati, 1997, Frascati Physics Series Vol. X (SIS Ufficio Pubblicazioni, Frascati, Italy, 1998), pp. $123-138$.

[4] I. Reichel, Ph.D. thesis, RWTH Aachen, 1998. 
[5] E. Keil, Nucl. Instrum. Methods Phys. Res. 188, 9-14 (1981).

[6] S. Myers, in Nonlinear Dynamic Aspects of Particle Accelerators (Ref. [1]), pp. 176-237.

[7] T. Chen, J. Irwin, and R. Siemann, in Proceedings of the 4th European Particle Accelerator Conference, edited by V. Suller and C. Petit-Jean-Genaz (World Scientific, Singapore, 1994), Vol. II, pp. 1356-1358.

[8] H. Burkhardt, in Proceedings of the 7th Advanced Beam Dynamics Workshop on Beam-Beam Issues for Multibunch, High-Luminosity Circular Colliders, Dubna, 1995 (ICFA, Dubna, 1996), pp. 22-28.

[9] H. Burkhardt et al., in Proceedings of the 5th European Particle Accelerator Conference, edited by V. Suller and C. Petit-Jean-Genaz (Institute of Physics, Bristol, Philadelphia, 1996), Vol. 2, pp. 1152-1154.

[10] I. Reichel, H. Burkhardt, and G. Roy, in Proceedings of the 1997 Particle Accelerator Conference, Vancouver, Canada, edited by M. Comyn, M. K. Craddock, M. Reiser, and J. Thomson (IEEE, Piscataway, NJ, 1998), Vol. 2, pp. 1819-1821.

[11] J. Jowett, in Proceedings of the 14th Advanced ICFA Beam
Dynamics Workshop on Beam Dynamics Issues for $e^{+} e^{-}$ Colliders, Frascati, 1997 (Ref. [3]), pp. 15-38.

[12] H. Burkhardt, in Proceedings of the 1997 Particle Accelerator Conference, Vancouver, Canada (Ref. [10]), pp. $1532-1536$.

[13] H. Burkhardt, in Proceedings of the 14th Advanced ICFA Beam Dynamics Workshop on Beam Dynamics Issues for $e^{+} e^{-}$Colliders, Frascati, 1997 (Ref. [3]), pp. 327-332.

[14] A. Zholents, in Proceedings of the 2nd Workshop on LEP Performance, edited by J. Poole (CERN, Geneva, 1992), pp. 387-390.

[15] W. Bialowons, F. Ridoutt, and K. Wittenburg, in Proceedings of the 4th European Particle Accelerator Conference (Ref. [7]), pp. 1628-1630.

[16] O. Gröbner, in Proceedings of the 3rd Workshop on LEP Performance, edited by J. Poole (CERN, Geneva, 1993), pp. 529-535.

[17] R. V. Servranckx, K. L. Brown, L. Schachinger, and D. Douglas, SLAC Report No. 285 UC-28 (A).

[18] H. Burkhardt, CERN SL Note No. 93-73 (OP).

[19] R. Kleiss and H. Burkhardt, Comput. Phys. Commun. 81, 372-380 (1994). 